\section{Preliminary data on ASP2215: tolerability and efficacy in acute myeloid leukemia patients}

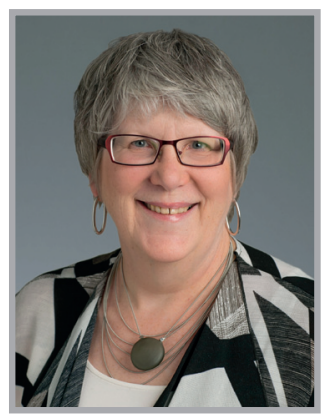

Claire Thom* speaks to Gemma Westcott, Commissioning Editor: Claire Thom joined Astellas in 2013 as the Therapeutic Area Head for Oncology in Global Development. In that role, she also serves as the STAR leader for Oncology for Astellas. Prior to Astellas, she spent 12 years with Takeda. Her last position was Senior Vice President, Portfolio Management, Drug Development Management and Medical Informatics and Strategic Operations within the Medical Division (the Division within Millennium responsible for oncology clinical drug development within Takeda). During her 4 years at Millennium, at various times, she had responsibility within the Medical Division for leading portfolio management, business operations (medical finance, annual and mid-range financial planning, space planning and operations, headcount resourcing, development goals process), clinical development operations (clinical operations, programming, data management, statistics, medical writing, clinical outsourcing), drug development management (project management), medical informatics (technology support for the division) and the strategic project management office for the division. Prior to joining Millennium, Claire Thom spent 18 months working in Osaka, Japan, during which she was responsible for developing the oncology strategy for Takeda that culminated in the acquisition of Millennium. Before going to Japan, she held positions of varying responsibility within the Takeda US development organization including the management of regulatory affairs, safety, biometrics and data management, clinical research and quality assurance. Claire Thom has particular expertise in organizational design and efficiency; she has successfully worked through integrations across multiple functions and redesigned business processes. She has a PharmD from University of Illinois (IL, USA) and over 20 years of pharmaceutical experience including positions in medical affairs and new product planning (over 11 years at Searle) and drug development (over 12 years at Takeda/Millennium).

Q How did you come to be in the role you are in today as Vice President of Global Oncology Development of Astellas?

I started my career as pharmacist working in hospital settings, then moved to the pharmaceutical industry setting about 25 years ago. I have worked in many different departments across several companies over the past 25 years, but I have gotten the most joy and satisfaction from working on new medicines to help patients with difficulty to treat diseases, such as oncology. It is magical to work with a team of professionals from different disciplines, using their collective skills and knowledge, to bring innovative science that is of value for patients, especially patients with cancer.

*Astellas Pharma Global Development, Inc., Northbrook, IL, USA

\section{KEYWORDS}

- acute myeloid leukemia • AML

- ASP2215 - Astellas • European Hematology Association Congress - FLT3 mutation 
Q Can you tell our readers what sparked your interest in cancer?

I am fascinated by the underlying science and biology of cancer and the medicines used to treat it. It is incredibly complex and there are continually new advances in the field. I am a lifelong learner and I suspect that we will never fully understand cancer in my lifetime, but we will make great advances.

Q What would you say has been your favorite moment in your career?

The day I realized that the XTANDI team was able to go from database lock on the PREVAIL study to regulatory approvals in USA, EU and Japan in less than 12 months. The amount of concerted teamwork and problem solving techniques required to achieve that was phenomenal and gives me pride whenever I speak of it and the impact it has on men with prostate cancer.

\section{Q Recent preliminary data from the} Phase I/II trial on the safety, tolerability \& efficacy of ASP2215 in patients with relapsed or refractory acute myeloid leukemia were

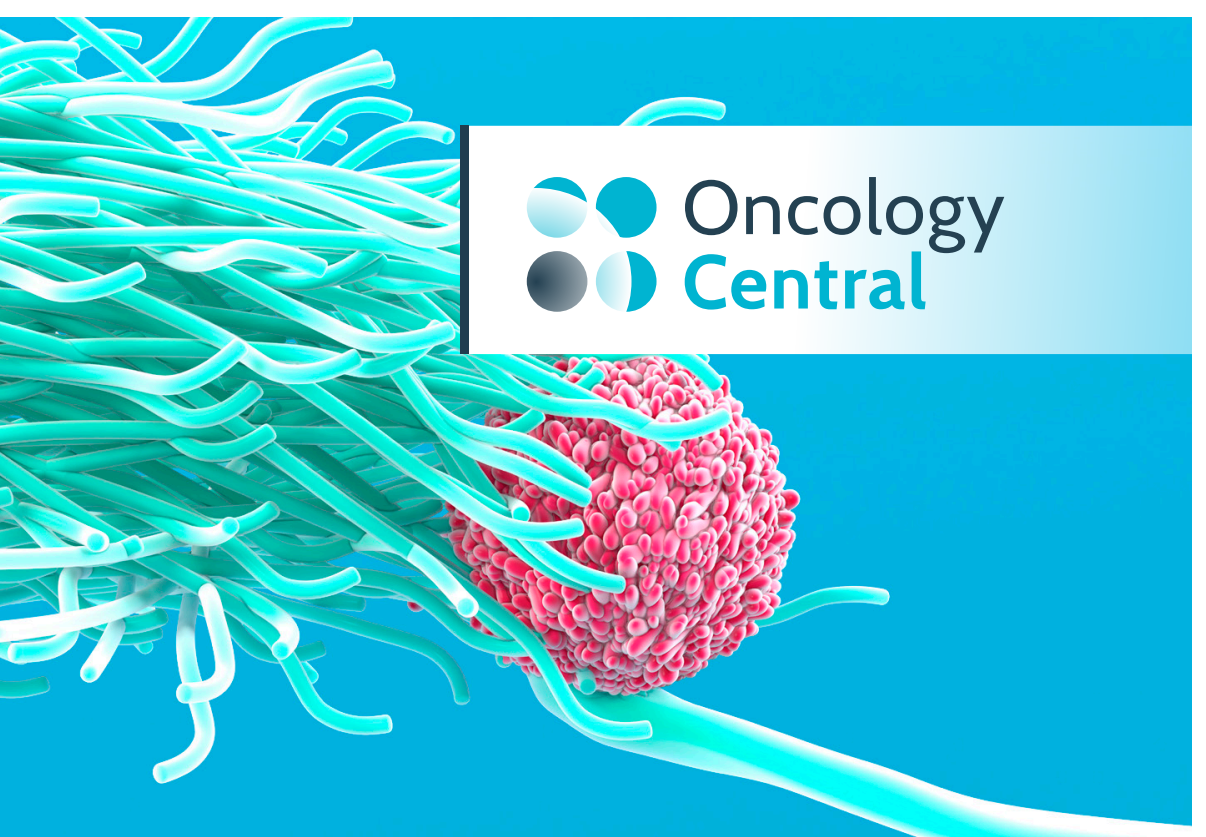

INTERACT AND LEARN
Join the conversation and influence the community

Join today

www. Oncology-Central.com presented at The American Society of Clinical Oncology Annual Meeting 2015 \& European Hematology Congress 2015. Can you tell us about ASP2215?

ASP2215 is a FLT3/AXL tyrosine kinase inhibitor [1]. It inhibits the function of FLT3 and AXL, molecules involved in the growth of cancer cells [1]. ASP2215 has demonstrated inhibitory activity against FLT3 internal tandem duplication (ITD) and tyrosine kinase domain (TKD), two common types of FLT3 mutations [1] that can occur in up to $30 \%$ of acute myeloid leukemia (AML) patients [1,2]. Both of these mutations are typically associated with poor patient outcomes [3]. As such, inhibiting the activities of this specific combination of molecule is of critical interest in treating this condition. Additionally, ASP2215 also inhibits AXL, a known resistance mechanism to chemotherapies in a variety of solid and hematological malignancies [1]. ASP2215 is currently under evaluation in a clinical setting for the treatment of relapsed or refractory (R/R) AML patients who are FLT3 positive.

Q What were the findings from the Phase I/II trial?

Preliminary data from the Phase I/II trial demonstrated a $57.5 \%$ overall response rate and a $47.2 \%$ composite complete remission (CR) rate $(\mathrm{CR}+\mathrm{CR}$ with incomplete platelet recovery + $\mathrm{CR}$ with incomplete hematologic recovery) in 106 patients with FLT3 mutations who received $80 \mathrm{mg}$ and higher doses [1]. A plasma inhibitory activity assay also confirmed sustained FLT3 inhibition consistently in patients receiving doses of $80 \mathrm{mg}$ and above [1]. Additional preliminary data from the Phase I/II trial first presented at the American Society of Clinical Oncology Annual Meeting 2015 and subsequently the European Hematology Congress 2015 also showed that the median duration of response was 18 weeks across all doses and median overall survival was approximately 27 weeks at $80 \mathrm{mg}$ and above in FLT3 mutation-positive patients [1]. As announced in June 2015, Phase I/II trial results appeared to show that ASP2215 is well tolerated at doses of between 20-300 mg, with $300 \mathrm{mg}$ being the maximum tolerated dose [1].

Q Can you tell our readers what implications these trial results could have for AML patients? The annual incidence rate of AML is estimated to be one in 33,000 to one in 25,000 in Europe [4]. In the USA, there are about 20,830 new cases 
of AML diagnosed annually [5], of which 30\% are FLT3 positive [1]. ASP2215 is currently being developed to treat patients with R/R AML who are FLT3 mutation positive. We believe that the preliminary data supports selecting patients who have R/R AML with FLT3 mutation-positive status [1].

\section{Q What would you say are the current} therapeutic challenges \& unmet needs in AML?

Drug resistance is an issue in the AML treatment paradigm [3]. The high morbidity and mortality rates associated with standard induction therapy for AML in the elderly combined with low complete response and survival rates make treating this specific population a challenge [6]. Additionally, the prognosis for older patients is also poor because intensive chemotherapy cannot be used in older patients due to the toxicity [6]. AML patient response to current salvage treatment is poor. Once relapsed, the disease is rapidly fatal [3].

\section{Q What is next for ASP2215 trialing/clinical development?}

ASP2215 is currently in Phase I/II development and a Phase III open label, multicenter, randomized study of ASP2215 versus salvage chemotherapy in patients with R/R AML with FLT3 mutation is planned to begin recruitment in the second half of 2015 .

Q Finally, where would you like to see the field of leukemia in 10 years' time?

We are excited about the concept of seeing more patients achieving initial cure through more tolerable regimens. Getting to bone marrow transplant is a key objective and if we can help make that happen, maybe with an outpatient rather than an inpatient regimen, we will have done our jobs well.

\section{Disclaimer}

The opinions expressed in this interview are those of the interviewee and do not necessarily reflect the views of Future Medicine Ltd.

\section{Financial \& competing interests disclosure}

C Thom acknowledges the support of Red Door Unlimited, a specialist Healthcare Communications agency, during the development of this article. C Thom is an employee of Astellas Pharma, Inc. C Thom has no other relevant affiliations or financial involvement with any organization or entity with a financial interest in or financial conflict with the subject matter or materials discussed in the manuscript apart from those disclosed.

No writing assistance was utilized in the production of this manuscript.

\section{References}

1 Perl A, Altman J, Cortes J et al. Results of a first in-human, Phase 1/2 trial of ASP2215, a selective, potent oral inhibitor of FLT3/AXL, in patients with relapsed or refractory acute myeloid leukaemia. Presented at: 20th Congress of the European Hematology Association. Vienna, Austria. 11-14 June 2015 (Abstract No. S798).
2 Levis M. FLT3 mutations in acute myeloid leukemia: what is the best approach in 2013? 2013(1), 220-226 (2013).

3 Grunwald MR, Levis MJ. FLT3 inhibitors for acute myeloid leukemia: a review of their efficacy and mechanisms of resistance. Int. J. Hematol. 97(6), 683-694 (2013).
5 National Cancer Institute. Adult acute myeloid leukemia treatment - for health professionals $\left(\mathrm{PDQ}^{\circledR}\right)$.

www.cancer.gov

6 American Cancer Society. Typical treatment of most types of acute myeloid leukemia (except acute promyelocytic M3). www.cancer.org 